\title{
An innovative approach to study the interaction between psychosocial profile and school drop-out
}

\author{
Francesca Mastorci ${ }^{1}$, Sara Macelloni ${ }^{2}$, Andrea Zavanella $^{2}$ and Alessandro Pingitore ${ }^{*}$ \\ ${ }^{1}$ Clinical Physiology Institute, CNR, Pisa, Italy \\ ${ }^{2}$ Formatica S.c.a.r.1., Pisa , Italy
}

\begin{abstract}
Adolescence is a transitional period from childhood to adulthood in which adolescents experienced physical, psychological, emotional, and social changes. It is both the healthiest period of the lifespan with respect to measurable parameters of psychophysical health, but at the same time, about half of the mental health problems in adults are estimated to have their onset during this critical time window. Although improving health and well-being of adolescents is a prominent global theme, health policies have responded in a one way approach, that is to evaluating only rates of diseases or negative health behaviours, such as addiction. To our knowledge, it is not available an integrated framework for psychosocial monitoring in youth that encompasses different dimensions of health in relationship with addiction vulnerability. Here, thanks to our experimental evidence collected in adolescents, we suggest a new approach in the field of mental health and prevention and treatment of substance use and abuse.
\end{abstract}

\section{Introduction}

For too long health adolescent has been defined by evaluating rates of diseases or negative health behaviours, such as substance abuse and addiction. Adolescence is, in fact, considered a period of contradictions. It is both the healthiest period of the lifespan with respect to measurable parameters of psychophysical health and the age in which behavioural risk factors can have crucial effects later in life, predisposing to chronic diseases in adulthood, originated from a combination of genetic, physiological, environmental, and behavioural factors. Not by chance, about half of the mental health problems in adults are estimated to have their onset in the adolescence [1], with profound implications for their social development and global economy.

Furthermore, from neurobiological perspective, the adolescent brain undergoes a phenomenon, called pruning, which makes it more efficient on the one hand, but more exposed to risk on the other. In the adolescent brain, this new balance changes the relationships between the major neural systems and their different neurotransmitters, influencing the adolescent's behaviour and making it more vulnerable to choices that have greater value in the short term. Therefore, a neurobiological mechanism may explain adolescent's attraction to discover new emotions and pleasures. For example, the increased activity of the dopaminergic system is responsible to reduce critical risk assessment skills, thus leading to expose adolescent to impulsive behaviours. In addition, prospective studies demonstrated that adolescents with recent negative life events, such as parental loss, chronic stress, social isolation, or low quality of life, increased levels and susceptibility of substance use and abuse [2].

This highlights the importance to develop preventive approaches and strategies contributing to support positive health from individual to community levels before it is too late.

In this context, strengthening connectedness with different social contexts, self-esteem, resilience and awareness of each adolescent can improve well-being that, in turn, is related to high mental and physical health, education attainment, and to reduction of unhealthy behaviours. At the same time, growing evidences indicate that adolescence is a dynamic and flexible period of knowledge and adaptation to target health interventions, so that adolescents can make positive lifestyle choices to enhance their well-being. However, to our knowledge, in order to define new prevention and treatment strategies, it is not available a framework for psychosocial monitoring in youth that encompasses different dimensions of health, including lifestyle habits, emotional status, social context. In this view, AVATAR project. "A new purpose for promotion and eVAluation of healTh and well-being Among healthy teenageRs" aims to promote well-being in adolescents thanks to a systemic and multi-stakeholder approach, which aims to create a network between the different figures and environments that revolve around the adolescent (family, school, peers, community) [3-5]. In particular, a section of this project is addressed to assess the potential risk factors of school drop-out and to prevent it through training/ educational and community programs. Specific objects of AVATARDropout are to enhance adolescent's aptitude skills (empowerment), to increase adaptive response to stimuli (resilience), that are intermediate steps for achieving psychophysical well-being and counter educational poverty. The proposed multifactorial framework is focused on the integration of four components of health-related well-being in adolescence: lifestyle habits (e.g diet and physical activity); emotional status (e.g self-esteem and stress level); social context (e.g relationship with peers and social network use), and risk factors (e.g substance use or abuse and addiction behaviour). Also, in order to recognise risk

${ }^{*}$ Correspondence to: Alessandro Pingitore, Clinical Physiology Institute, CNR, Via Moruzzi, 1, 56124 Pisa, Italy, E-mail: pingi@ifc.cnr.it

Key words: adolescent, drop-out, addiction, health, well-being

Received: September 20, 2020; Accepted: September 28, 2020; Published: October 01,2020 
subjects and identify the strength and the fragile characteristics of each adolescent to potentiate the first ones and to change or improve the others through the application of personalised educational programs, AVATAR project has developed a Personalised Well-Being Index (PWBI) [4]. Our preliminary and unpublished data collected by a sample of 150 adolescents (14-16 years old) who had dropped out of school, showed that there was no correlation between psychosocial profile, in term of PWBI, and risk factors, such as substance use and abuse, social media addiction, and eating disorders. Our results are in line with the general opinion not based on objective data, which states that adolescents in drop-out, report a high frequency of risk behaviours, especially with regard to substance use (more than $50 \%$ in our sample), therefore considering addiction one of the main risk factors for early school leaving. However, despite this high prevalence of addiction behaviours, school drop-out was not associated with low well-being, as often thought, but on the contrary, was characterized by a higher perception of well-being, especially in drug users. This result, although preliminary, shows how the relationship between wellbeing and addiction in adolescents is not so clear, but rather requires a careful analysis in all its variables, with particular attention to the type of substance, first use and possible causes, in order to define preventive and health promotion strategies. Evidence to clarify the contribution of psychosocial profile to alterations in well-being and health-related quality of life, and its association with drug use in adolescents is also needed. In order to change unhealthy individual behaviours, it is necessary to create environmental conditions that favour a healthy lifestyle, through a transversal approach to risk factors and social settings in which adolescents live. The innovative framework suggested by AVATAR-Dropout resides in creating a link between the different figures that have the ability to affect health and well-being in adolescents, identifying the risks, but also opportunities in the different contexts. Whereas experimental evidence indicates that adolescents at risk for substance abuse are often characterized by reduced executive functioning, low behavioural and emotional control, higher levels of stress response to stimuli, poor decision-making and impulsiveness [6], the prevention of addiction cannot fail to start from these psychological and neurobiological aspects. For this reason, the main pillars of the AVATAR approach are aimed at: strengthening health education, through a comprehensive health education curriculum that focuses on increasing students' understanding of healthy behaviours and modifying risk factors; support health services and schools, concentrating on the prevention and early identification of psychological issues that could evolve toward overt disease; create a healthful environment, concerned with the physical and psychosocial setting including school, family and community. Therefore, to foster well-being among adolescents is a priority, also in the field of addiction, to bridge the gap between psychosocial determinants and school dropout, from both the health and education perspective, making health and well-being more centred on adolescent's needs and fragility.

\section{References}

1. Kessler RC, Avenevoli S, McLaughlin KA, Greif Green J, Lakoma MD, et al. (2012) Lifetime co-morbidity of DSM-IV disorders in the US National Comorbidity Survey Replication Adolescent Supplement (NCS-A). Psychol Med 42: 1997-2010. [Crossref]

2. Barrett A, Turner R (2006) Family structure and substance use problems in adolescence and early adulthood: examining explanations for the relationship. Addiction 101: 109120. [Crossref]

3. Trivellini G, Doveri C, Mastorci F, Bastiani L, Cappa C, et al. (2018) Innovative webbased tool for promoting well-being among healthy adolescents: An implementation protocol. J Transl Sci 5: 1-5.

4. Mastorci F, Bastiani L, Doveri C, Trivellini G, Casu A, et al. (2020) Adolescent Health A Framework for Developing an Innovative Personalized Well-Being Index. Front Pediatr 8: 181. [Crossref]

5. Mastorci F, Bastiani L, Trivellini G, Doveri C, Vassalle C, et al. (2020) A new integrated approach for adolescent health and well-being: the AVATAR project. Health Qual Life Outcomes 18: 77.

6. Baler RD, Volkow ND (2006) Drug addiction: the neurobiology of disrupted selfcontrol. Trends Mol Med 12: 559-566. [Crossref]

Copyright: (C2020 Mastorci F. This is an open-access article distributed under the terms of the Creative Commons Attribution License, which permits unrestricted use, distribution, and reproduction in any medium, provided the original author and source are credited. 